\title{
BMJ Open Prognostic accuracy of different disseminated intravascular coagulation criteria in critically ill adult patients: a protocol for a systematic review and meta-analysis
}

Jumpei Yoshimura, Kazuma Yamakawa, Akira Kodate, Mari Kodate, Satoshi Fujimi

To cite: Yoshimura J, Yamakawa K, Kodate A, et al. Prognostic accuracy of different disseminated intravascular coagulation criteria in critically ill adult patients: a protocol for a systematic review and meta-analysis. BMJ Open 2018;8:e024878. doi:10.1136/ bmjopen-2018-024878

- Prepublication history for this paper is available online. To view these files, please visit the journal online (http://dx.doi org/10.1136/bmjopen-2018024878).

Received 19 June 2018 Revised 16 0ctober 2018 Accepted 25 October 2018
Check for updates

(c) Author(s) (or their employer(s)) 2018. Re-use permitted under CC BY-NC. No commercial re-use. See rights and permissions. Published by BMJ.

Division of Trauma and Surgical Critical Care, Osaka General Medical Center, Osaka, Japan

Correspondence to

Dr Kazuma Yamakawa;

k.yamakawa0911@gmail.com

\section{ABSTRACT}

Introduction Disseminated intravascular coagulation

(DIC) is a common and serious condition that can lead to poor outcomes in critically ill patients. To make a correct diagnosis and improve the outcome of patients with DIC, several organisations have put forward DIC scoring systems. However, which criteria is the best to use for diagnosing DIC remains a continuing controversy even though many studies have been conducted to validate the diagnostic accuracy of each DIC scoring system.

Methods and analysis We will conduct a systematic review and meta-analysis of the diagnostic accuracy of DIC criteria for the prediction of mortality in critically ill adult patients. The primary objective is to assess the predictive values of the DIC criteria of Japanese Association for Acute Medicine, International Society on Thrombosis and Haemostasis, Japanese Ministry of Health and Welfare, Korean Society on Thrombosis and Hemostasis and Chinese Diagnostic Scoring System for 28-day mortality. We will search electronic bibliographic databases such as MEDLINE, EMBASE and Cochrane Central Register of Controlled Trials. Two reviewers will independently screen titles and abstracts, perform full article reviews and extract study data. We will report study characteristics and assess methodological quality using the Quality of Diagnostic Accuracy Studies-II tool. If pooling is appropriate, we will compute parameter estimates using bivariate random-effects and hierarchical summary receiver operating characteristic models to produce summary receiver operating curves, summary operating points (pooled sensitivity and specificity) and $95 \%$ confidence regions around the summary operating points. Clinical and methodological subgroup and sensitivity analyses will be performed to explore heterogeneity.

Ethics and dissemination This systematic review will help physicians diagnose DIC accurately and improve their clinical practice in critically ill settings. Approval from an ethics committee is not required. The findings will be disseminated through publication in a peer-reviewed journal.

PROSPERO registration number CRD42017079350.

\section{Strengths and limitations of this study}

- This systematic review will be the first report to answer the clinical question of which disseminated intravascular coagulation (DIC) criteria can most accurately predict mortality and be useful in overall populations of critically ill patients and patients with sepsis.

- The supposed major limitation in this review will be that we only assess the prognostic values and not the diagnostic values of the DIC criteria, due to the lack of a gold standard for DIC diagnosis.

- We will carry out a systematic review using appropriate methodologies and quality assessment tools according to the Grading of Recommendations Assessment, Development and Evaluation approach.

- We believe that the results will be directly useful in helping clinicians to better select DIC criteria based on the best available evidence.

\section{INTRODUCTION}

Disseminated intravascular coagulation (DIC) is a disorder frequently seen in critically ill patients, especially with severe sepsis or multiple trauma ${ }^{1}$ that may lead to severe bleeding and organ dysfunction. Because mortality is higher in patients with DIC than in those without DIC, ${ }^{1}$ several organisations have put forward DIC scoring systems with the aim of improving the outcome of patients with DIC. The Japanese Ministry of Health and Welfare (JMHW) proposed a criteria for the diagnosis of DIC in $1976 .{ }^{2}$ The criteria involved the evaluation of global coagulation tests, underlying diseases and clinical symptoms. Thereafter, the subcommittee of the International Society on Thrombosis and Haemostasis (ISTH) proposed a scoring system for overt and non-overt DIC in 2001. However, it was easy for the diagnosis to be made too late based on the JMHW or the 
ISTH criteria in settings of critical illness and especially for sepsis patients. Thus, the Japanese Association for Acute Medicine (JAAM) proposed another DIC scoring system that was aimed at making an early diagnosis of DIC for patients on acute medicine. ${ }^{4}$ In addition, other criteria for DIC were proposed, those of the Korean Society on Thrombosis and Hemostasis (KSTH) and the Chinese DIC Scoring System (CDSS) ${ }^{56}$

The controversy over which criteria are the best to use for diagnosing DIC continues even though many studies have been conducted over the years to validate the diagnostic accuracy of each DIC scoring system. ${ }^{5-12}$ Japanese and Italian clinical practice guidelines for DIC management recommend the use of either the JMHW, ISTH or the JAAM criteria while the British guideline recommends the use of the ISTH criteria. ${ }^{13-15}$ Although the guidelines do not offer consistent recommendations about diagnosing DIC, no meta-analysis has been conducted so far to compare the prognostic performance among the several available DIC criteria. Thus, we will conduct a systematic review and meta-analysis of the prognostic accuracy of several DIC scoring systems in critically ill adult patients.

\section{METHODS AND ANALYSIS \\ Protocol}

This review adopts recommendations on the conduct and reporting of systematic reviews and meta-analyses outlined by the Preferred Reporting Items for Systematic Reviews and Meta-Analyses (PRISMA) statement, ${ }^{16-18}$ the Meta-Analysis of Observational Studies in Epidemiology proposal $^{19}$ and the Cochrane Diagnostic Test Accuracy Working Group. ${ }^{20}$

\section{Focused review questions}

Primary Objective To determine and compare the prognostic values of ISTH overt DIC criteria and non-overt DIC criteria, JMHW DIC criteria, JAAM DIC criteria, KSTH DIC criteria and CDSS DIC criteria for mortality in critically ill adult patients.

Secondary Objective To determine which criteria are superior for the prediction of mortality, especially focused on adult patients with sepsis diagnosed by Sepsis-1, Sepsis-2 or Sepsis-3. ${ }^{21-23}$

\section{Types of studies}

We will include all studies that assess the diagnostic accuracy of any DIC criteria noted above in adult critically ill patients. Diagnostic accuracy studies are typically of a delayed cross-sectional design. However, we will also include randomised controlled trials, cohort studies and case-control studies. Included studies should have sufficient information to build a $2 \times 2$ contingency table (true and false, positive and negative). Articles with experimental animals, narrative reviews, correspondence, case reports, expert opinions and editorials will be excluded.

\section{Types of participants}

We will include studies evaluating critically ill adult patients aged 18 years or older. Since 'critical illness' is somewhat poorly defined we will include critical illnesses whose definitions are generally accepted, such as acute respiratory distress syndrome, sepsis and systemic inflammatory response syndrome in this review. These will include participants from different clinical settings, such as emergency departments, hospital wards and intensive care units. We will exclude all studies investigating animals, those predominantly comprising neonates or postcardiac surgical, heart failure or perioperative patients and those comprising healthy participants as controls.

\section{Studied tests}

The index tests will be the ISTH overt DIC criteria ${ }^{3}$ and non-overt DIC criteria, ${ }^{3}$ JMHW DIC criteria, ${ }^{2}$ JAAM DIC criteria, ${ }^{4}$ KSTH DIC criteria ${ }^{5}$ and CDSS DIC criteria. ${ }^{6}$ A summary of DIC criteria is shown in table 1. In case DIC criteria are assessed more than once, the data evaluated at the earliest time point will be used as the index test. Cut-off values of fibrin-related markers, antithrombin, protein $\mathrm{C}$ and thrombin-antithrombin complex in the ISTH overt/non-overt DIC criteria will be defined according to the individual studies.

\section{Reference standards}

The reference standard will be 28-day mortality, which is one of the standard outcome measures in critical care settings. In case multiple mortality outcomes are reported or 28-day mortality is not available, the mortality outcome closest to 28-day will be handled as the reference standard.

\section{Exclusion criteria}

We will exclude studies from which we cannot obtain or calculate the true and false and positive and negative rates from the text, appendices or after contacting the main authors; abstracts that do not provide enough information for the assessment of the methodological quality; and duplicates or subcohorts of already published cohorts.

\section{Search strategy}

We will search the following databases for relevant studies: MEDLINE (via PubMed), EMBASE and the Cochrane Central Register of Controlled Trials. We have developed a search strategy using a combination of keywords and medical subject headings (MeSH)/EMTREE terms, which are '('disseminated intravascular coagulation' OR DIC) AND ('International Society on Thrombosis and Haemostasis' OR ISTH OR 'Japanese Ministry of Health and Welfare' OR JMHW OR 'Japanese Association for Acute Medicine' OR JAAM OR ‘Korean Society on Thrombosis and Hemostasis' OR KSTH OR 'Chinese Diagnostic Scoring System' OR CDSS OR overt OR non-overt)'. We will screen the reference lists of all relevant papers for additional studies. We will also contact relevant authors for further details and information on ongoing/ unpublished trials. Our MEDLINE search strategy will be adapted appropriately for searches in the other two databases. No language restrictions will be applied. Only article beyond 1976 will be reviewed because none of the scoring systems became available before 1976 . 


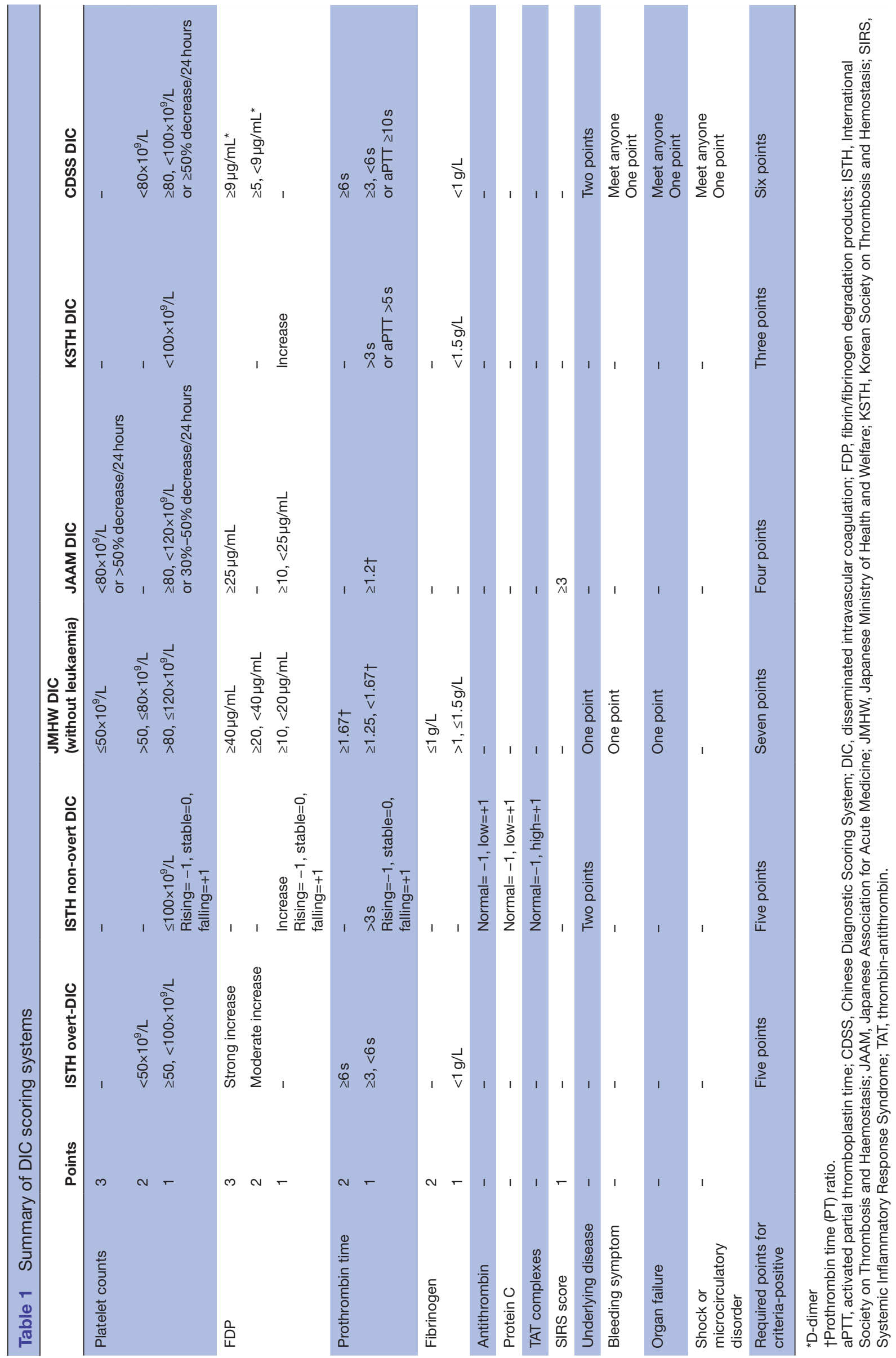

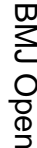




\section{Citation management and screening}

Citations will be stored and duplicates will be removed using EndNote software (Thomson Reuters, Toronto, Ontario, Canada). Studies will be screened initially according to title and abstract by two authors (JY, MK) independently and those not meeting the criteria will be discarded. Disagreements will be resolved by discussion and referral to a third author (KY), if necessary. After this initial stage, the full text of all remaining studies will be reviewed by two authors (JY, MK) independently for inclusion or exclusion in the final study. Disagreements will be resolved in the same way as the initial screening. We will use Covidence software (Covidence; Melbourne, Australia) in this screening process. We will document the study selection process in a PRISMA flow diagram.

\section{Data extraction}

Two authors (JY, AK) will extract the study characteristics from each included study, including data on the assessment of quality and the investigation of heterogeneity and transfer that information into a study-specific format. Adjudication by a third author (KY) will be used if necessary. We will cross-tabulate the numerical information from the index test results (positive or negative) in $2 \times 2$ tables against the target disorder (positive or negative) and we will present the results as tables. Efforts will be made to contact the authors of primary studies to provide missing data where necessary.

\section{Assessment of risk of bias}

The quality of the included studies will be independently assessed by two authors (JY, AK) and verified by a third $(\mathrm{KY})$, if necessary. The study quality of each article will be reported according to the Quality Assessment of Diagnostic Accuracy Studies (QUADAS-2) tool. ${ }^{24}$ The presence of spectrum, threshold, disease progression and verification bias (partial or differential) will be specifically assessed. For each domain, we will assign a judgement regarding the risk of bias as high, low or unclear. We will attempt to contact the trial corresponding author for clarification when insufficient detail is reported to assess the risk of bias.

\section{Data synthesis}

We will present the results graphically by plotting estimates of sensitivities and specificities (both with 95\% CI) in a Forest plot and in a receiver operating characteristic (ROC) space to visually assess the between-study variability. We will use Review Manager software, RevMan 5.3 (Nordic Cochrane Centre, Cochrane Collaboration) to document the descriptive analyses.

We will pool studies only if they share a common threshold, are conducted in the same or similar setting and show sufficient clinical homogeneity. For the meta-analysis, we will fit a summary ROC curve using a bivariate random-effects model and derive summary accuracy indices (sensitivity and specificity) and corresponding likelihood ratios using the MIDAS module for STATA software, V.14.0. Also, we will estimate positive predictive value and negative predictive value which are more useful clinically. We will plot the $95 \%$ confidence ellipse and prediction region around averaged accuracy estimates in the ROC space. We will generate a nomogram, which is a user-friendly graphical depiction of positive predictive value and negative predictive value by prevalence.

\section{Assessment of heterogeneity}

We will investigate heterogeneity initially by visual examination of Forest plots of sensitivities and specificities and through visual examination of individual study results in the ROC space. Statistical heterogeneity will be evaluated informally from Forest plots of the study estimates and more formally using the $\chi^{2}$ test ( $p$ value $<0.1$, significant heterogeneity) and $\mathrm{I}^{2}$ statistic $\left(\mathrm{I}^{2}>50 \%=\right.$ significant heterogeneity).

\section{Assessment of publication biases}

If a sufficient number of studies are identified, we will investigate publication bias by Deeks' funnel plot. We will interpret this analysis with caution given the lack of statistical power of this test and the absence of consensus about adequate methods to detect publication bias in reviews of diagnostic test accuracy.

\section{Sensitivity and subgroup analysis}

We will examine the robustness of the meta-analyses by conducting sensitivity analyses and excluding studies according to different components of the QUADAS-2 tool for assessing the risk of bias. Our primary analysis will include all studies, and sensitivity analysis will exclude those studies with high risk of bias or with important concerns about potential applicability.

If sufficient studies are available, we will undertake subgroup analyses to explore the sources of potential heterogeneity in sensitivity and specificity. Meta-regression analysis and subgroup analysis will be performed using the following as covariates: sepsis or not, trauma or not, year of publication, country, prevalence of DIC $(<50 \%$ or $\geq 50 \%)$, baseline mortality risk, sample size $(<100$ or $\geq 100)$, types of fibrin-related markers, and cut-off values of fibrin-related markers.

\section{Interpretation and summary of findings}

A major focus of reviews of diagnostic test accuracy is to obtain summary estimates of test accuracy. However, knowing that a test has a high sensitivity, for instance, does not tell us whether the test will have much effect on the patient, nor does it tell us that using this test in practice will be beneficial for the patient or cost-effective. The Grading of Recommendations Assessment, Development and Evaluation (GRADE) approach for diagnostic tests has now been developed, which provides guidance on how to translate accuracy data into a recommendation involving patient-important outcomes. ${ }^{25}$ We will apply the GRADE approach to rate the quality of the evidence. 


\section{Patient and public involvement}

Patients and the public were not involved in the design of this protocol.

\section{DISCUSSION}

DIC is a common and serious condition that can lead to poor outcomes in critically ill patients. ${ }^{1}$ Although several guidelines for DIC management have been published to improve its clinical practice ${ }^{13-15}$ the evidence for diagnosing DIC accurately is limited. Therefore, physicians still have difficulty diagnosing and managing DIC in clinical settings and there is a need for properly designed, well-conducted reports to determine which criteria should be used to assess DIC.

There will be potential major limitations in this review. First, we will only assess the prognostic values and not the diagnostic values of the DIC criteria. Initially, the efficacy of the diagnostic test accuracy should be evaluated on sensitivity and specificity for the presence of the disease. Because of the lack of a gold standard for DIC diagnosis, we cannot properly assess the diagnostic rates of the DIC criteria. Alternatively, we will compare the prognostic values of each of the DIC criteria in this review. Second, several interventions, such as transfusion and anticoagulant therapy, can affect coagulation parameters and subsequently, the DIC score. As there may be no predefined protocol for diagnostic time points, we will use the data for assessment of DIC criteria that was evaluated at the earliest time point as the index test to minimise the influence of clinical exposure in this review. Third, the variety of study populations can influence the heterogeneity of the predictive value of the DIC criteria. Thus, we will perform subgroup analyses focused on sepsis and trauma, which are the major causes of DIC in critically ill patients.

This meta-analysis will be the first report to reveal the characteristics of several DIC criteria for the prediction of mortality and may be useful in assessing overall populations of critically ill patients and sepsis patients. We will carry out a systematic review of the diagnostic test accuracy of several DIC criteria for predicting mortality using appropriate methodologies and quality assessment tools according to the GRADE approach. Thus, we believe that the results will be directly useful in helping clinicians to better select DIC criteria based on the best available evidence.

\section{ETHICS AND DISSEMINATION}

Approval from an ethics committee is not required since this systematic review will use publicly available data without directly involving human participants. Our findings will be presented at relevant scientific conferences and disseminated through publication in a peer-reviewed journal.

Contributors KY was a guarantor and contributed to the conception of the study. The manuscript protocol was drafted by JY and KY and was revised by SF. The search strategy was developed by all of the authors and will be performed by JY and MK, who will also independently screen the potential studies. JY and AK will independently extract data from the included studies, assess the risk of bias and complete the data synthesis. KY will arbitrate in cases of disagreement and ensure the absence of errors. All authors approved the publication of this protocol.

Funding The authors have not declared a specific grant for this research from any funding agency in the public, commercial or not-for-profit sectors.

Competing interests None declared.

Patient consent for publication Not required.

Provenance and peer review Not commissioned; externally peer reviewed.

Open access This is an open access article distributed in accordance with the Creative Commons Attribution Non Commercial (CC BY-NC 4.0) license, which permits others to distribute, remix, adapt, build upon this work non-commercially, and license their derivative works on different terms, provided the original work is properly cited, appropriate credit is given, any changes made indicated, and the use is non-commercial. See: http://creativecommons.org/licenses/by-nc/4.0/.

\section{REFERENCES}

1. Levi $\mathrm{M}$, ten Cate $\mathrm{H}$. Disseminated intravascular coagulation. $N$ Engl J Med Overseas Ed 1999;341:586-92.

2. Kobayashi N, Maekawa T, Takada M, et al. Criteria for diagnosis of DIC based on the analysis of clinical and laboratory findings in 345 DIC patients collected by the research committee on DIC in Japan. Bibl Haematol 1983;49:265-75.

3. Taylor FB, Toh CH, Hoots WK, et al. Towards definition, clinical and laboratory criteria, and a scoring system for disseminated intravascular coagulation. Thromb Haemost 2001;86:1327-30.

4. Gando S, Saitoh D, Ogura H, et al. Natural history of disseminated intravascular coagulation diagnosed based on the newly established diagnostic criteria for critically ill patients: results of a multicenter, prospective survey. Crit Care Med 2008;36:145-50.

5. Lee JH, Song JW, Song KS. Diagnosis of overt disseminated intravascular coagulation: a comparative study using criteria from the International Society versus the Korean Society on Thrombosis and Hemostasis. Yonsei Med J 2007;48:595-600.

6. Thrombosis and hemostasis study group in hematology branch of the Chinese Medical Association. Chinese experts' consensus on the diagnosis and treatment of disseminated intravascular coagulation (2012 edition). Chin J Haematol 2012;33:978-9.

7. Toh $\mathrm{CH}$, Downey C. Performance and prognostic importance of a new clinical and laboratory scoring system for identifying non-overt disseminated intravascular coagulation. Blood Coagul Fibrinolysis 2005;16:69-74.

8. Egi M, Morimatsu H, Wiedermann CJ, et al. Non-overt disseminated intravascular coagulation scoring for critically ill patients: the impact of antithrombin levels. Thromb Haemost 2009;101:696-705.

9. Iwai K, Uchino S, Endo A, et al. Prospective external validation of the new scoring system for disseminated intravascular coagulation by Japanese Association for Acute Medicine (JAAM). Thromb Res 2010;126:217-21.

10. Hayakawa M, Gando S, Hoshino H. A prospective comparative study of three sets of criteria for disseminated intravascular coagulation: ISTH criteria vs Japanese criteria. Clin Appl Thromb Hemost 2007;13:65-72.

11. Gando S, Saitoh D, Ogura H, et al. A multicenter, prospective validation study of the Japanese Association for Acute Medicine disseminated intravascular coagulation scoring system in patients with severe sepsis. Crit Care 2013;17:R111.

12. Wang $\mathrm{M}$, Kou H, Deng J, et al. Retrospective evaluation of new chinese diagnostic scoring system for disseminated intravascular coagulation. PLoS One 2015;10:e0129170.

13. Wada $\mathrm{H}$, Asakura $\mathrm{H}$, Okamoto $\mathrm{K}$, et al. Expert consensus for the treatment of disseminated intravascular coagulation in Japan. Thromb Res 2010;125:6-11.

14. Di Nisio M, Baudo F, Cosmi B, et al. Diagnosis and treatment of disseminated intravascular coagulation: guidelines of the Italian Society for Haemostasis and Thrombosis (SISET). Thromb Res 2012;129:e177-e184.

15. Levi M, Toh $\mathrm{CH}$, Thachil J, et al. Guidelines for the diagnosis and management of disseminated intravascular coagulation. British Committee for standards in haematology. Br J Haematol 2009;145:24-33.

16. Moher D, Liberati A, Tetzlaff J, et al. Preferred reporting items for systematic reviews and meta-analyses: the PRISMA Statement. Open Med 2009;3:e123-30. 
17. Liberati A, Altman DG, Tetzlaff J, et al. The PRISMA statement for reporting systematic reviews and meta-analyses of studies that evaluate health care interventions: explanation and elaboration. $J$ Clin Epidemiol 2009;62:e1-e34.

18. Moher D, Shamseer L, Clarke M, et al. Preferred reporting items for systematic review and meta-analysis protocols (PRISMA-P) 2015 statement. Syst Rev 2015;4:1.

19. Stroup DF, Berlin JA, Morton SC, et al. Meta-analysis of observational studies in epidemiology: a proposal for reporting. Meta-analysis Of Observational Studies in Epidemiology (MOOSE) group. JAMA 2000;283:2008-12.

20. Leeflang MM, Deeks JJ, Gatsonis C, et al. Systematic reviews of diagnostic test accuracy. Ann Intern Med 2008;149:889-97.

21. American College of Chest Physician/Society of Critical Care Medicine Consensus Conference. definitions for sepsis and organ failure and guidelines for the use of innovative therapies in sepsis. Crit Care Med 1992;20:864-74.

22. Levy MM, Fink MP, Marshall JC, et al. 2001 SCCM/ESICM/ACCP/ ATS/SIS International Sepsis Definitions Conference. Intensive Care Med 2003;29:530-8.

23. Singer M, Deutschman CS, Seymour CW, et al. The third international consensus definitions for sepsis and septic shock (Sepsis-3). JAMA 2016;315:801-10.

24. Whiting PF, Rutjes AW, Westwood ME, et al. QUADAS-2: a revised tool for the quality assessment of diagnostic accuracy studies. Ann Intern Med 2011;155:529-36.

25. Hsu J, Brożek JL, Terracciano L, et al. Application of GRADE: making evidence-based recommendations about diagnostic tests in clinical practice guidelines. Implement Sci 2011;6:6-62. 